\title{
Obesity Epidemic: Is Its Impact on Total Joint Arthroplasty Underestimated? An Analysis of National Trends
}

\author{
Jaiben George MBBS, Alison K. Klika MS, Suparna M. Navale MS, \\ MPH, Jared M. Newman MD, Wael K. Barsoum MD, Carlos A. Higuera MD
}

Published online: 4 January 2017

(C) The Association of Bone and Joint Surgeons (B) 2017

\begin{abstract}
Background Obesity is a well-established risk factor for total joint arthroplasty (TJA) and a number of complications including prosthetic joint infection. The annual changes in the prevalence of obesity among primary, revision, and infected TJA has not been studied at a national level. Given the higher costs of complications of TJA, it is important to understand the association of obesity with the annual trends of revision and infected TJA.

Questions/purposes (1) Is the prevalence of obesity increasing among patients undergoing THA/TKA? (2) Is the prevalence of obesity increasing among patients undergoing revision THA/TKA? (3) Is the prevalence of obesity increasing among patients with infected THA/ TKA?
\end{abstract}

One of the authors certifies that he (WKB), or a member of his immediate family, has or may receive payments or benefits, during the study period an amount of USD 100,001 to USD 1,000,000 from Stryker (Kalamazoo, MI, USA); an amount of USD 100,001 to USD 1,000,000 from Exactech (Gainesville, FL, USA); an amount of less than USD 10,000 from Zimmer (Warsaw, IN, USA); an amount of less than USD 10,000 from Custom Orthopaedic Solutions (Cleveland, OH, USA); an amount of USD 100,001 to USD 1,000,000 from KEF Healthcare (Westminster, CO, USA); an amount of less than USD 10,000 from iVHR (Cleveland, OH, USA); an amount of less than USD 10,000 from PeerWell (San Francisco, CA, USA); and has received research support from Zimmer (Warsaw, IN, USA), DJO (Vista, CA, USA), and Orthosensor (Dania Beach, FL, USA). One of the authors $(\mathrm{CAH})$ or a member of his immediate family, has or may receive payments or benefits, during the study period, an amount of less than USD 10,000 from KCI (San Antonio, TX, USA); an amount of less than USD 10,000 from Zimmer Biomet (Warsaw, IN, USA); an amount of less than USD 10,000 from Covance (Princeton, NJ, USA); an amount of less than USD 10,000 from Pfizer (New York, NY, USA); and has received research support funding from Stryker (Kalamazoo, MI, USA), Myoscience (Fresno, CA, USA), CD

Diagnostics (Claymont, DE, USA), KCI (San Antonio, TX, USA),
Methods Annual volumes of primary, revision, and infected THA and TKA from 1998 to 2011 were obtained from the Nationwide Inpatient Sample. Using mathematical equations, the prevalence of obesity was estimated from relative risks and national obesity prevalence. National obesity prevalence was obtained from public health sources and the relative risk estimates were obtained from previously published meta-analyses and population-based studies. Annual prevalence of obesity was obtained by dividing the number of obese primary/revision/infected procedures in each year by the total number of corresponding procedures in that year. Annual changes in the prevalence of obesity were analyzed using linear regression.

Results The prevalence of obesity is increasing among patients undergoing THA (1998: 60,264 of 154,337 [39\%],

OREF (Rosemont, IL, USA), and Pacira (Parsippany, NJ, USA). All ICMJE Conflict of Interest Forms for authors and Clinical Orthopaedics and Related Research ${ }^{\mathbb{B}}$ editors and board members are on file with the publication and can be viewed on request. This work was performed at the Cleveland Clinic, Cleveland, $\mathrm{OH}$, USA.

Electronic supplementary material The online version of this article (doi:10.1007/s11999-016-5222-4) contains supplementary material, which is available to authorized users.

J. George, A. K. Klika ( $₫)$, J. M. Newman, W. K. Barsoum, C. A. Higuera

Department of Orthopedic Surgery, Cleveland Clinic, 9500

Euclid Avenue, A40, Cleveland, OH 44195, USA

e-mail: klikaa@ccf.org

S. M. Navale

Department of Epidemiology and Biostatistics, Case Western

Reserve University, Cleveland, OH, USA 
2011: 160,241 of 305,755 [52\%], increase of $1.05 \% / y$ ear [confidence interval $\{\mathrm{CI}\}, 0.95 \%-1.15 \%], \mathrm{p}<0.001)$ and TKA (1998: 143,681 of 251,309 [57\%], 2011: 448,712 of 644,243 [70\%], increase of $0.97 \% / y e a r$ [CI, $0.87 \%-$ $1.07 \%$ ], $\mathrm{p}<0.001)$. There was an increasing prevalence of obesity with THA revisions (1998: 16,322 of 34,139 [48\%], 2011: 33,304 of 54,453 [61\%], increase of $1.04 \%$ / year [CI, $0.94 \%-1.15 \%], \mathrm{p}<0.001)$ and in TKA revisions (1998: 16,837 of 26,539 [63\%], 2011: 52,151 of 69,632 [75\%], increase of $0.89 \% / y e a r$ [CI, $0.79 \%-0.99 \%$ ], p < $0.001)$. There was an increasing prevalence of obesity with THA infections (1998: 2068 of 3018 [69\%], 2011: 6856 of 8687 [79\%], increase of $0.80 \% / y e a r$ [CI, $0.71 \%-0.89 \%$ ], p $<0.001)$ and in TKA infections (1998: 3563 of 4684 [76\%], 2011: 14,178 of 16,774 [85\%], increase of $0.65 \%$ / year [CI, $0.57 \%-0.73 \%$ ], $\mathrm{p}<0.001$ ).

Conclusions The prevalence of obesity has increased in patients undergoing primary, revision, and infected TJA in United States. The obesity epidemic appears to be related to the growing trends of revision and infection after TJA. With the obesity rates expected to grow further, the revision and infection burden associated with obesity may increase in the future.

Level of Evidence Level II, prognostic study.

\section{Introduction}

Obesity has been described as an epidemic in the United States [33]. Approximately one-third of the US adult population is estimated to be obese, representing a serious public health issue [38]. With knee and hip osteoarthritis being more prevalent in obese patients, it is not surprising that the rise in obesity rates is expected to be associated with an increase in use of total joint arthroplasty (TJA) in the United States [2, 20, 27, 36]. Obese patients are also at a higher risk for complications after TJA, including infection, wound complications, and revision arthroplasty $[8,14,18,40,42,43]$.

The prevalence of obesity among patients undergoing TJA at a national level is yet to be studied. To the best of our knowledge, the only study that evaluated the national trends was performed by Odum et al. [37] using the Nationwide Inpatient Sample (NIS) database. Although they identified an annual increase in the obesity prevalence in patients undergoing TKA in the United States, the prevalence of obesity among patients undergoing TKA reported in their study (approximately 20\%) was much lower than what has been previously reported in studies from a single institution (50\%-60\%) and even lower than the prevalence seen in the general population (approximately $30 \%$ ) [11, 19, 38, 39]. Even if one assumes the risk of undergoing TJA to be equal in obese and nonobese individuals, the prevalence of obesity in patients undergoing TJA would be expected to be at least comparable to the general population. The lower estimates using the NIS database are possibly the result of the inaccuracies of obesity coding in administrative databases [12, 29]. With the advancements in surgical techniques and implant designs, the success rates of TJA are anticipated to improve $[23,44]$. However, previous studies have reported an increasing infection burden and a relatively stable revision burden of TJA in the United States [20, 22]. The association of obesity toward the growing infection burden and stable revision burden has not been studied. Given the greater costs of revision surgeries, it is important to understand the impact obesity has on revision and infection burden after TJA [7, 16].

We therefore asked: (1) Is the prevalence of obesity increasing among patients undergoing THA/TKA? (2) Is the prevalence of obesity increasing among patients undergoing revision THA/TKA? (3) Is the prevalence of obesity increasing among patients with infected THA/TKA?

\section{Materials and Methods}

The prevalence of obesity in the US population was used in conjunction with the estimates of risk for undergoing TJA in obese individuals to determine the prevalence of obesity among TJA from 1998 to 2011. Similarly, the prevalence of obesity in revision and infected TJA was obtained using the obesity prevalence data and the corresponding risk of revision and infection in obese individuals. The annual trends in the procedure volumes, revision burden, and infection burden were studied and the association with obesity was evaluated. Analyses were performed for knees and hips separately.

The annual number of TKAs and THAs was obtained from the NIS database [35]. As a result of the stratified sampling format of the NIS, all frequencies and analyses were converted to national estimates using weights and accounting for the complex sample design. The International Classification of Disease, 9th Revision, Clinical Modification (ICD-9-CM) codes were used to identify primary TKA and THA (81.54 and 81.51 , respectively). The ICD-9-CM codes for revision TKA were 80.06 and $81.55,00.80-84$ [6] and for revision THA were 80.05 and $81.53,00.70-73$ [5]. Infected TJA was defined as any primary/revision TJA with the ICD-9 code 996.66 in the same hospitalization episode [22].

The prevalence of obesity among primary, revision, and infected TJA was calculated as described subsequently. TKA and THA calculations were performed separately using the general equations shown subsequently. The number of obese individuals undergoing a primary TJA in a given year, $t$, can be expressed as: 
$\mathrm{TJA}_{\mathrm{t}}^{\text {obese }}=\frac{\left(\mathrm{TJA}_{\mathrm{t}}^{\mathrm{total}} \times \mathrm{O}_{\mathrm{t}} \times \mathrm{J}^{\mathrm{TJA}}\right)}{\left(\mathrm{O}_{\mathrm{t}} \times \mathrm{J}^{\mathrm{TJA}}\right)+\left(100-\mathrm{O}_{\mathrm{t}}\right)}$

where $\mathrm{TJA}_{\mathrm{t}}^{\text {total }}$ represents the total number of primary TJAs for year $t, \mathrm{O}_{\mathrm{t}}$ represents the national obesity prevalence (in percentage) for year $t$, and $\mathrm{J}^{\mathrm{TJA}}$ is the risk of undergoing a TJA in obese patients compared with nonobese individuals.

The number of obese individuals undergoing a revision TJA in a given year, $t$, can be expressed as:

$$
\mathrm{RTJA}_{\mathrm{t}}^{\text {obese }}=\frac{\mathrm{RTJ}_{\mathrm{t}}^{\text {total }} \times \mathrm{O}_{\mathrm{t}} \times \mathrm{J}^{\mathrm{TJA}} \times \mathrm{R}^{\mathrm{TJA}}}{\left(\mathrm{O}_{\mathrm{t}} \times \mathrm{J}^{\mathrm{TJA}} \times \mathrm{R}^{\mathrm{TJA}}\right)+\left(100-\mathrm{O}_{\mathrm{t}}\right)}
$$

where $\mathrm{RTJA}_{\mathrm{t}}^{\text {total }}$ represents the total number of revision TJAs for year $t$ and $\mathrm{R}^{\mathrm{TJA}}$ is the risk for undergoing a revision procedure in obese patients undergoing TJA compared with nonobese patients undergoing TJA.

The number of obese individuals with an infected TJA in a given year, $\mathrm{t}$, can be expressed as:

$$
\mathrm{ITJA}_{\mathrm{t}}^{\text {obese }}=\frac{\mathrm{ITJA}_{\mathrm{t}}^{\text {total }} \times \mathrm{O}_{\mathrm{t}} \times \mathrm{J}^{\mathrm{TJA}} \times \mathrm{I}^{\mathrm{TJA}}}{\left(\mathrm{O}_{\mathrm{t}} \times \mathrm{J}^{\mathrm{TJA}} \times \mathrm{I}^{\mathrm{TJA}}\right)+\left(100-\mathrm{O}_{\mathrm{t}}\right)}
$$

where $\operatorname{ITJA}_{\mathrm{t}}^{\text {total }}$ represents the total number of infected TJAs (primary or revision) for year $t$ and $\mathrm{I}^{\mathrm{TJA}}$ is the risk of acquiring a deep infection in obese patients undergoing TJA compared with nonobese patients undergoing TJA.

The relative risks were assumed to remain constant throughout the study period. The relative risks used in this study were obtained from previously published studies. A literature search was performed in PubMed/Medline (1995-2015) using a combination of keywords: obesity, meta-analysis, arthroplasty, population, and risk. Because the results of different studies show considerable variations, only meta-analyses or population-based studies were considered to obtain the risk estimates. The search yielded one population-based study reporting the risk of TJA in obese patients (Bourne et al. [2]), one meta-analysis reporting the outcomes after TKA in obese patients (Kerkhoffs et al. [18]), and two meta-analyses reporting the outcomes after THA in obese patients (Haverkamp et al. [14] and Liu et al. [24]). Because the meta-analysis by Haverkamp et al. [14] involved more patients, the estimates of their study were used. Relative risks for THA $\left(\mathrm{J}^{\mathrm{THA}}\right)$ and TKA $\left(\mathrm{J}^{\mathrm{TKA}}\right)$ were assigned the values 2.86 and 5.96, respectively. These values were obtained from the study by Bourne et al. [2], which evaluated the risks for undergoing TJA in obese individuals based on a national joint registry and population data. The values of revision TKA $\left(\mathrm{R}^{\mathrm{TKA}}\right)$ and revision THA $\left(\mathrm{R}^{\text {THA }}\right)$ were 1.30 (95\% confidence interval [CI], 1.02-1.67) and 1.43 (95\% CI, 1.10-1.86), respectively. The values of infected THA $\left(\mathrm{I}^{\mathrm{THA}}\right)$ and TKA $\left(\mathrm{I}^{\mathrm{TKA}}\right)$ were 3.4 (95\% CI, 2.09-5.53) and 2.38 (95\% CI, 1.28-4.55), respectively. Except for revision THA, all of the other values $\left(\mathrm{I}^{\mathrm{THA}}, \mathrm{I}^{\mathrm{TKA}}, \mathrm{R}^{\mathrm{TKA}}\right)$ were obtained from meta-analyses reporting odds ratio for the respective complications [14, 18]. Because a meta-analysis reporting the risk for revision from all causes for THA was not available in the literature, the input for revision THA was calculated from pooling the results of multiple studies obtained from the studies included in the meta-analyses by Haverkamp et al. [14] and Liu et al. [1, 15, 17, 24, 26, 30]. The fixed-effects forest plot showing the pooled odds ratio is given in Appendix 1 (Supplemental materials are available with the online version of $C O R R^{\circledR}$.). Although the formulas used in this study were designed to use the relative risk, this statistic could not be obtained from casecontrol studies [14, 18]. Zhang et al. [46] demonstrated that the relative risks and odds ratios tend to be similar when the prevalence of an outcome is low with correction desirable only when the odds ratio is $>2.5$ (or $<0.5$ ) and the prevalence of the outcome was $>10 \%$. Most studies have reported the prevalence of revision to be less than $10 \%$, whereas the reported prevalence of infection is usually $1 \%$ to $2 \%[14,15,21,22,26,30,31]$. Therefore, correction was not performed for the odds ratios included in this study.

Obesity prevalence was defined as the percentage of the population with a body mass index (BMI) of $\geq 30 \mathrm{~kg} / \mathrm{m}^{2}$ according to the World Health Organization classification [45]. Annual prevalence of obesity (term $\mathrm{O}_{t}$ in the equations) from 1998 to 2011 was obtained from the Behavioral Risk Factor Surveillance System (BRFSS), a nationwide cross-sectional health survey run by the Centers for Disease Control and Prevention, which collects data from more than 400,000 adults annually [3]. Although the BRFSS does not take the actual measures of height and weight of respondents, it is the largest survey in the United States and provides the annual obesity prevalence $[3,4]$. The infection burden and revision burden were defined in agreement with other studies [20, 22]. The revision burden in a given year was defined as the total number of revision TJAs divided by the total number of TJAs (primary + revision). The infection burden was defined as the number of infected TJAs among all TJAs (primary + revision). The revision and infection burdens associated with obesity were calculated by dividing the number of obese patients undergoing a revision or infected TJA by the total number of TJAs (primary + revision) for any given year.

\section{Statistical Analysis}

The annual growth in prevalence of obesity among primary, revision, and infected TJAs was studied using linear regression analysis. Annual changes in the revision and infection burden were assessed using a simple linear regression model. 
The slope or coefficient of the linear regression model provided the changes/year of prevalence and burden. A positive slope denotes an increasing prevalence or burden, whereas a negative slope denotes a decreasing prevalence or burden. If the slope was not statistically significant, the prevalence or burden was considered to be stable across the study period. Sensitivity analyses were performed to understand the variations in the results with changes in relative risks (Appendix 1). A p value of 0.05 was used as the cutoff for statistical significance.

\section{Results}

The prevalence of obesity is increasing among patients undergoing THA (1998: 60,264/154,337 [39\%], 2011: $160,241 / 305,755$ [52\%], increase of $1.05 \% / y e a r$ [CI, 0.95\%-1.15\%], $\mathrm{p}<0.001)$ and TKA (1998: 143,681 of 251,309 [57\%], 2011: 448,712 of 644,243 [70\%], increase of $0.97 \% / y e a r$ [CI, $0.87 \%-1.07 \%$ ] $\mathrm{p}<0.001$ ) (Table 1 ; Fig. 1).

There was an increasing prevalence of obesity in revision THA (1998: 16,322 of 34,139 [48\%], 2011: 33,304 of 54,453 [61\%], increase of $1.04 \% / y e a r$ [CI, $0.94 \%-1.15 \%$ ], $\mathrm{p}<0.001)$ and in revision TKA (1998: 16,837 of 26,539 [63\%], 2011: 52,151 of 69,632 [75\%], increase of $0.89 \% /$ year [CI, 0.79\%-0.99\%], p < 0.001) (Table 2; Fig. 2). The revision burden declined in THA (1998: 34,139 of 188,476 [18.1\%], 2011: 54,453 of 360,208 [15.1\%], decrease of $0.39 \% / y e a r$ [CI, $-0.50 \%$ to $-0.24 \%], \mathrm{p}<0.001)$ but remained unchanged in TKA (1998: 26,539 of 277,848 [9.6\%], 2011: 69,632 of 713,875 [9.8\%], decrease of $0.03 \% /$ year [CI, $-0.11 \%$ to $0.04 \%$ ], $\mathrm{p}=0.356$ ) (Table 3 ;

Table 1. Prevalence of obesity among patients undergoing total joint arthroplasty

\begin{tabular}{lll}
\hline Year & THA & TKA \\
\hline 1998 & $39 \%(60,264 / 154,337)$ & $57 \%(143,681 / 251,309)$ \\
1999 & $41 \%(64,374 / 156,706)$ & $59 \%(155,596 / 262,687)$ \\
2000 & $42 \%(68,564 / 164,458)$ & $60 \%(168,468 / 281,534)$ \\
2001 & $43 \%(79,475 / 184,646)$ & $61 \%(191,813 / 313,618)$ \\
2002 & $45 \%(89,529 / 201,167)$ & $63 \%(219,051 / 350,122)$ \\
2003 & $46 \%(92,569 / 201,544)$ & $64 \%(242,647 / 379,719)$ \\
2004 & $46 \%(104,706 / 225,900)$ & $64 \%(277,406 / 431,485)$ \\
2005 & $48 \%(114,069 / 237,645)$ & $66 \%(327,279 / 497,419)$ \\
2006 & $49 \%(111,766 / 228,382)$ & $67 \%(330,567 / 496,077)$ \\
2007 & $51 \%(127,224 / 251,882)$ & $68 \%(374,213 / 550,161)$ \\
2008 & $51 \%(141,092 / 276,528)$ & $69 \%(421,543 / 615,716)$ \\
2009 & $51 \%(145,991 / 284,708)$ & $69 \%(425,970 / 620,192)$ \\
2010 & $52 \%(157,038 / 301,798)$ & $69 \%(455,255 / 656,635)$ \\
2011 & $52 \%(160,241 / 305,755)$ & $70 \%(448,712 / 644,243)$ \\
\hline
\end{tabular}

Fig. 3). Interestingly, there was no change in the revision burden associated with obese patients in THA (1998: 16,322 of 188,476 [8.7\%], 2011: 33,304 of 360,208 [9.2\%], decrease of $0.04 \%$ year [CI, $-0.10 \%$ to $0.02 \%$ ], $\mathrm{p}=0.214$ ) and an increase in the obese revision burden in TKA (1998: 16,837 of 277,848 [6.1\%], 2011: 52,151 of 713,875 [7.3\%], increase of $0.06 \%$ year [CI, $0.01 \%,-0.11 \%$ ], $\mathrm{p}=0.020$ ). In the meantime, there was a decrease in revision burden from nonobese patients in THA (1998: 17,817 of 188,476 [9.5\%], 2011: 21,149 of 360,208 [5.9\%], decrease of $0.33 \% / y e a r$ [CI, $-0.41 \%$ to $-0.26 \%$ ], $\mathrm{p}<0.001$ ) and TKA (1998: 9702 of 277,848 [3.5\%], 2011: 17,481 of 713,875 [2.4\%], decrease of $0.09 \% / y e a r$ [CI, $-0.12 \%$ to $-0.06 \%$ ], p $<0.001)$. The annual growth in revision burden in obese patients was higher than that in nonobese patients for both THA (difference in slopes $=0.30 \% /$ year [CI, 0.21-0.39], $\mathrm{p}$

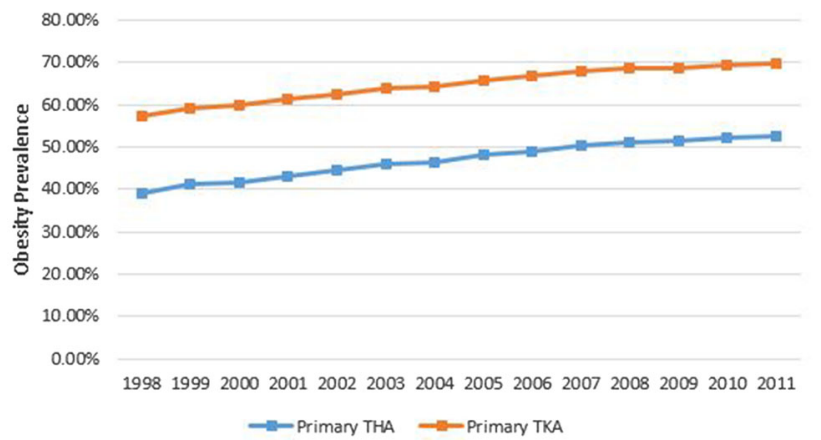

Fig. 1 The figure shows the annual trends in the prevalence of obesity in primary THA (slope $=1.05 \%$ year [CI, $0.95 \%-1.15 \%$ ], $\mathrm{p}<0.001$ ) and TKA (slope $=0.97 \% /$ year $[\mathrm{CI}, 0.87 \%-1.07 \%$ ], $\mathrm{p}<0.001)$ performed in the United States.

Table 2. Prevalence of obesity among patients undergoing revision total joint arthroplasty

\begin{tabular}{lll}
\hline Year & THA & TKA \\
\hline 1998 & $48 \%(16,322 / 34,139)$ & $63 \%(16,837 / 26,539)$ \\
1999 & $50 \%(16,639 / 33,327)$ & $65 \%(18,171 / 27,792)$ \\
2000 & $51 \%(18,495 / 36,584)$ & $66 \%(18,356 / 27,832)$ \\
2001 & $52 \%(21,521 / 41,437)$ & $67 \%(20,871 / 31,066)$ \\
2002 & $53 \%(21,221 / 39,725)$ & $68 \%(23,370 / 34,127)$ \\
2003 & $55 \%(20,554 / 37,475)$ & $70 \%(24,708 / 35,445)$ \\
2004 & $55 \%(22,157 / 40,092)$ & $70 \%(27,662 / 39,480)$ \\
2005 & $57 \%(22,791 / 40,057)$ & $71 \%(32,119 / 44,963)$ \\
2006 & $58 \%(22,475 / 38,873)$ & $72 \%(32,538 / 45,069)$ \\
2007 & $59 \%(24,607 / 41,468)$ & $73 \%(35,968 / 48,977)$ \\
2008 & $60 \%(27,208 / 45,471)$ & $74 \%(43,053 / 58,308)$ \\
2009 & $60 \%(26,297 / 43,770)$ & $74 \%(41,925 / 56,630)$ \\
2010 & $61 \%(29,752 / 48,931)$ & $75 \%(48,928 / 65,577)$ \\
2011 & $61 \%(33,304 / 54,453)$ & $75 \%(52,151 / 69,632)$ \\
\hline
\end{tabular}




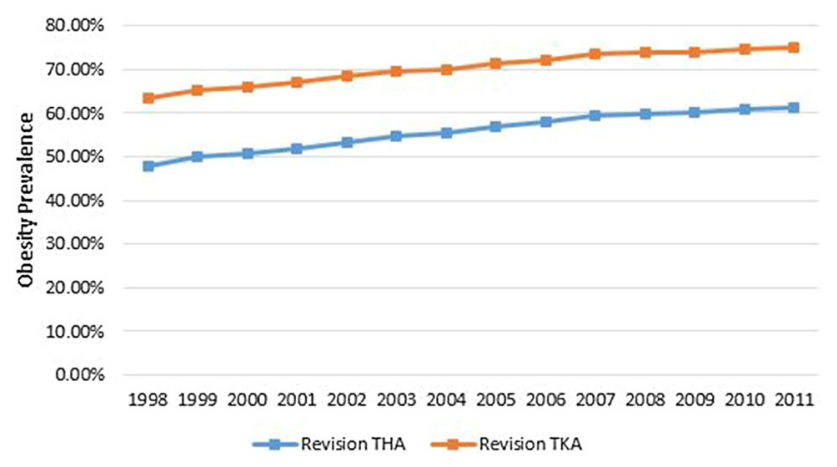

Fig. 2 The figure shows the annual trends in the prevalence of obesity in revision THA (slope $=1.04 \%$ year [CI, $0.94 \%-1.15 \%$ ], $\mathrm{p}<0.001$ ) and TKA (slope $=0.89 \% /$ year $[\mathrm{CI}, 0.79 \%-0.99 \%$ ], $\mathrm{p}<0.001)$ performed in the United States.

$<0.001$ ) and TKA (difference in slopes $=0.15 \% /$ year $[\mathrm{CI}$, $0.10-0.20], \mathrm{p}<0.001)$.

There was an increasing prevalence of obesity in infected THA (1998: 2068 of 3018 [69\%], 2011: 6856 of 8687 [79\%], increase of $0.80 \% / y e a r$ [CI, $0.71 \%-0.89 \%$ ], p $<0.001)$ and in infected TKA (1998: 3563 of 4684 [76\%], 2011: 14,178 of 16,774 [85\%], increase of $0.65 \%$ year [CI, $0.57 \%-0.73 \%$ ], $\mathrm{p}<0.001$ ) (Table 4; Fig. 4). The infection burden increased in THA (1998: 3018 of 188,476 [1.6\%], 2011: 8687 of 360,208 [2.4\%], increase of $0.05 \% / y e a r$ [CI, 0.04\%-0.06\%], $\mathrm{p}<0.001)$ and TKA (1998: 4684 of 277,848 [9.6\%], 2011: 16,774 of 713,875 [9.8\%], increase of $0.04 \% / y e a r$ [CI, $0.03 \%-0.05 \%$ ] $\mathrm{p}<0.001$ ) (Table 5 ; Fig. 5). In the meantime, there was an increase in the infection burden associated with obese patients in THA (1998: 2068 of 188,476 [1.1\%], 2011: 6856 of 360,208 [1.9\%], increase of $0.05 \% /$ year [CI, $0.04 \%-0.06 \%$ ], p < 0.001 ) and TKA (1998: 3563 of 277,848 [1.3\%], 2011: 14,178 of 713,875 [2.0\%], increase of $0.05 \% / y e a r$ [CI, $0.03 \%-0.06 \%$ ], $\mathrm{p}<0.001)$. Interestingly, there was no change in the infection burden associated with nonobese patients in THA (1998: 950 of 188,476 [0.5\%], 2011: 1831 of 360,208 [0.5\%], decrease of $0.01 \% / y e a r$ [CI, $-0.01 \%$ to $0.00 \%], \mathrm{p}=0.085)$ and a slight decrease in the nonobese infection burden in TKA (1998: 1121 of 277,848 [0.4\%], 2011: 2596 of 713,875 [0.4\%], decrease of $0.01 \% / y e a r$ [CI, $-0.01 \%$ to $0.00 \%], \mathrm{p}=0.002$ ). The annual growth in infection burden in obese patients was higher than that in nonobese patients for both THA (difference in slopes = 0.06\%/year [CI, 0.05-0.07], $\mathrm{p}<0.001$ ) and TKA (difference in slopes $=0.05 \% /$ year [CI, 0.04-0.06], $\mathrm{p}<0.001$ ).

\section{Discussion}

The obesity epidemic in the United States is believed to be at least partly responsible for the growing demand for TJA

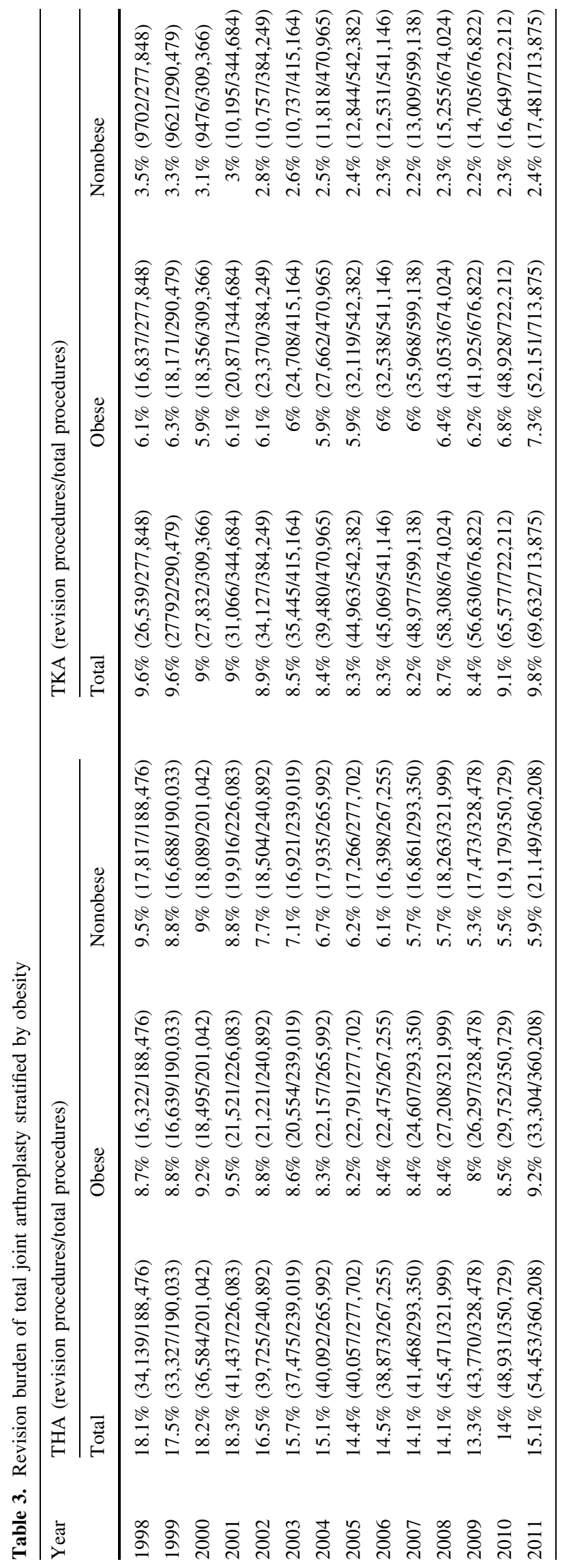




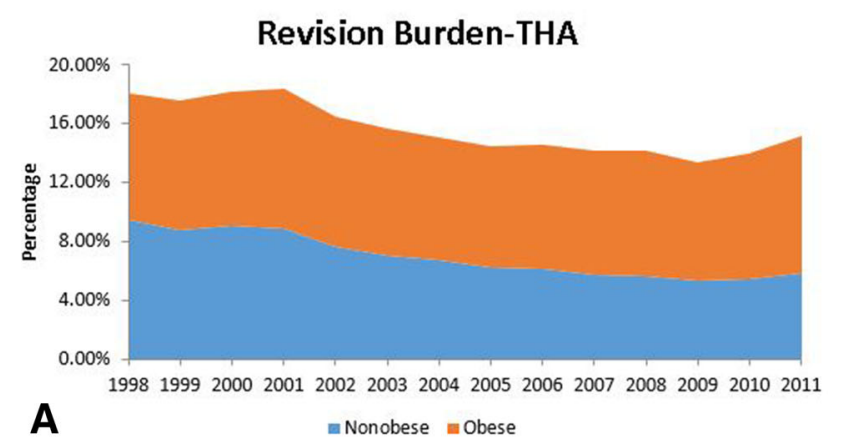

Fig. 3A-B The figure shows the annual trends in the revision burden of THA (A) and TKA (B) in the United States stratified by obesity. The annual growth in revision burden in obese patients was higher than that in nonobese patients for both THA (difference in slopes =

Table 4. Prevalence of obesity among infected total joint arthroplasty

\begin{tabular}{llr}
\hline Year & THA & \multicolumn{1}{l}{ TKA } \\
\hline 1998 & $69 \%(2068 / 3018)$ & $76 \%(3563 / 4684)$ \\
1999 & $70 \%(2194 / 3119)$ & $78 \%(3901 / 5029)$ \\
2000 & $71 \%(2556 / 3608)$ & $78 \%(4087 / 5239)$ \\
2001 & $72 \%(2851 / 3960)$ & $79 \%(4675 / 5922)$ \\
2002 & $73 \%(3355 / 4586)$ & $80 \%(5669 / 7094)$ \\
2003 & $74 \%(3468 / 4669)$ & $81 \%(6626 / 8199)$ \\
2004 & $75 \%(3958 / 5305)$ & $81 \%(7332 / 9043)$ \\
2005 & $76 \%(3886 / 5124)$ & $82 \%(8694 / 10,593)$ \\
2006 & $77 \%(4347 / 5681)$ & $83 \%(8811 / 10,665)$ \\
2007 & $78 \%(5006 / 6448)$ & $84 \%(9538 / 11,422)$ \\
2008 & $78 \%(5359 / 6872)$ & $84 \%(11,300 / 13,487)$ \\
2009 & $78 \%(5253 / 6721)$ & $84 \%(10,933 / 13,027)$ \\
2010 & $79 \%(5898 / 7497)$ & $84 \%(13,477 / 15,982)$ \\
2011 & $79 \%(6856 / 8687)$ & $85 \%(14,178 / 16,774)$ \\
\hline
\end{tabular}

[9, 25]. With more frequent complications reported in obese individuals, the increasing obesity prevalence may result in a dramatic increase in expensive revision surgeries [18]. In the present study, we aimed to study the annual changes in the prevalence of obesity among primary, revision, and infected TJA using data estimated from NIS data, obesity prevalence in the general population, and relative risk estimates for TJA and its complications.

The primary challenge of this study was in obtaining reliable risk estimates for various procedures in obese patients compared with nonobese patients. Because a single study reporting all the risk values was not available, the values were obtained from multiple studies, which in itself can act as a potential source of error as a result of underlying variations in the study populations. To minimize the errors, the inputs were obtained from population-based studies, meta-analyses, or by compiling the results of

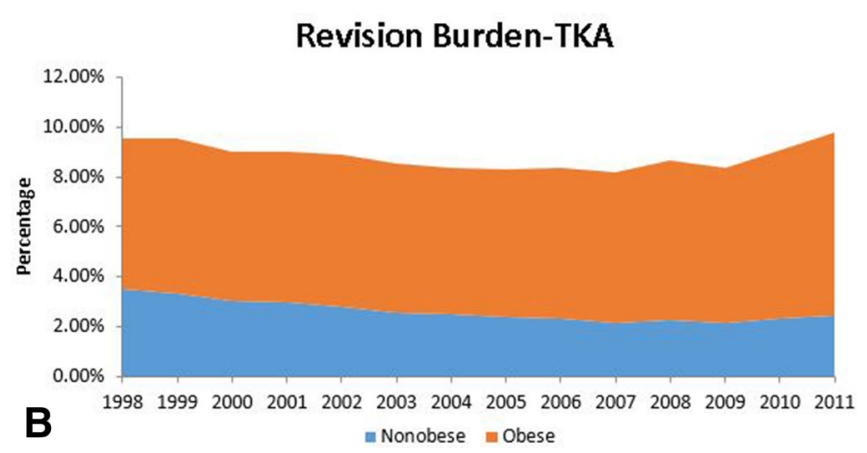

0.30\%/year [CI, 0.21-0.39], $\mathrm{p}<0.001$ ) and TKA (difference in slopes $=0.15 \% /$ year $[\mathrm{CI}, 0.10-0.20], \mathrm{p}<0.001)$.

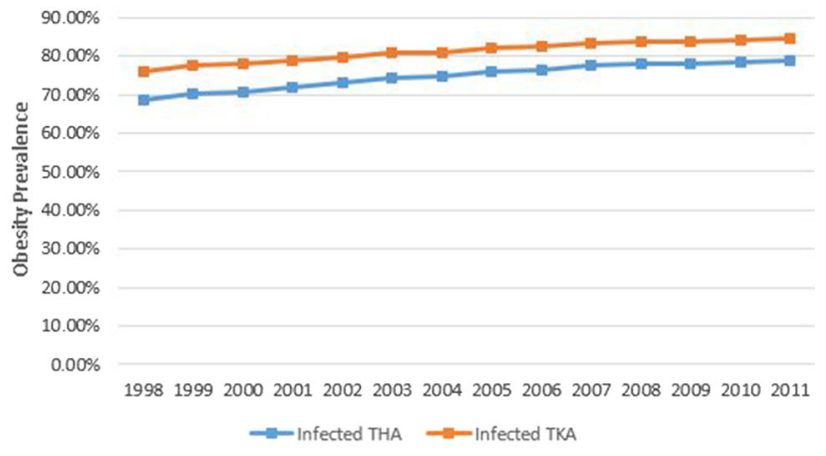

Fig. 4 The figure shows the annual trends in the prevalence of obesity in infected THA (slope $=0.80 \% /$ year [CI, $0.71 \%-0.89 \%$ ], $\mathrm{p}<0.001$ ) and TKA (slope $=0.65 \% /$ year $[\mathrm{CI}, 0.57 \%-0.73 \%$ ], $\mathrm{p}<0.001)$ performed in the United States

multiple studies. The use of estimates from such large studies is expected to provide the most reliable results and is a strength of this article. However, it should be noted that various studies have conflicting reports on the risk of infection/revision in obese patients, and the impact of obesity is less clear with respect to THA [40, 42]. Moreover, the risk estimates for TJA were obtained from a study based on a Canadian population and may be different from that seen in the US population, especially if there is a selection bias against performing TJA in obese patients [2]. Additionally, the relative risk values can change during the period of study and might influence the study results (Appendix 1). Although it is possible that gender, age, and comorbidities can potentially modify the association of obesity with TJA, stratified risks based on gender, age, and each comorbidity for every procedure were not available and, hence, they could not be assessed. In the current study, obesity was considered as a single category (BMI $\geq 30 \mathrm{~kg} /$ $\mathrm{m}^{2}$ ), although higher rates of complications are expected in morbidly obese and super obese patients [8, 43]. Infection burden was analyzed for all TJAs (primary and revision) 
Table 5. Infection burden of total joint arthroplasty stratified by obesity

\begin{tabular}{|c|c|c|c|c|c|c|}
\hline \multirow[t]{2}{*}{ Year } & \multicolumn{3}{|c|}{ THA (infected procedures/total procedures) } & \multicolumn{3}{|c|}{ TKA (infected procedures/total procedures) } \\
\hline & Total & Obese & Nonobese & Total & Obese & Nonobese \\
\hline 1998 & $1.6 \%(3018 / 188,476)$ & $1.1 \%(2068 / 188,476)$ & $0.5 \%(950 / 188,476)$ & $1.7 \%(4684 / 277,848)$ & $1.3 \%(3563 / 277,848)$ & $0.4 \%(1121 / 277,848)$ \\
\hline 1999 & $1.6 \%(3119 / 190,033)$ & $1.2 \%(2194 / 190,033)$ & $0.5 \%(925 / 190,033)$ & $1.7 \%(5029 / 290,479)$ & $1.3 \%(3901 / 290,479)$ & $0.4 \%(1128 / 290,479)$ \\
\hline 2000 & $1.8 \%(3608 / 201,042)$ & $1.3 \%(2556 / 201,042)$ & $0.5 \%(1052 / 201,042)$ & $1.7 \%(5239 / 309,366)$ & $1.3 \%(4087 / 309,366)$ & $0.4 \%(1152 / 309,366)$ \\
\hline 2001 & $1.8 \%(3960 / 226,083)$ & $1.3 \%(2851 / 226,083)$ & $0.5 \%(1109 / 226,083)$ & $1.7 \%(5922 / 344,684)$ & $1.4 \%(4675 / 344,684)$ & $0.4 \%(1247 / 344,684)$ \\
\hline 2002 & $1.9 \%(4586 / 240,892)$ & $1.4 \%(3355 / 240,892)$ & $0.5 \%(1231 / 240,892)$ & $1.8 \%(7094 / 384,249)$ & $1.5 \%(5669 / 384,249)$ & $0.4 \%(1425 / 384,249)$ \\
\hline 2003 & $2 \%(4669 / 239,019)$ & $1.5 \%(3468 / 239,019)$ & $0.5 \%(1201 / 239,019)$ & $2 \%(8199 / 415,164)$ & $1.6 \%(6626 / 415,164)$ & $0.4 \%(1573 / 415,164)$ \\
\hline 2004 & $2 \%(5305 / 265,992)$ & $1.5 \%(3958 / 265,992)$ & $0.5 \%(1347 / 265,992)$ & $1.9 \%(9043 / 470,965)$ & $1.6 \%(7332 / 470,965)$ & $0.4 \%(1711 / 470,965)$ \\
\hline 2005 & $1.8 \%(5124 / 277,702)$ & $1.4 \%(3886 / 277,702)$ & $0.4 \%(1238 / 277,702)$ & $2 \%(10593 / 542,382)$ & $1.6 \%(8694 / 542,382)$ & $0.4 \%(1899 / 542,382)$ \\
\hline 2006 & $2.1 \%(5681 / 267,255)$ & $1.6 \%(4347 / 267,255)$ & $0.5 \%(1334 / 267,255)$ & $2 \%(10665 / 541,146)$ & $1.6 \%(8811 / 541,146)$ & $0.3 \%(1854 / 541,146)$ \\
\hline 2007 & $2.2 \%(6448 / 293,350)$ & $1.7 \%(5006 / 293,350)$ & $0.5 \%(1442 / 293,350)$ & $1.9 \%(11422 / 599,138)$ & $1.6 \%(9538 / 599,138)$ & $0.3 \%(1884 / 599,138)$ \\
\hline 2008 & $2.1 \%(6872 / 321,999)$ & $1.7 \%(5359 / 321,999)$ & $0.5 \%(1513 / 321,999)$ & $2 \%(13487 / 674,024)$ & $1.7 \%(11300 / 674,024)$ & $0.3 \%(2187 / 674,024)$ \\
\hline 2009 & $2 \%(6721 / 328,478)$ & $1.6 \%(5253 / 328,478)$ & $0.4 \%(1468 / 328,478)$ & $1.9 \%(13027 / 676,822)$ & $1.6 \%(10933 / 676,822)$ & $0.3 \%(2094 / 676,822)$ \\
\hline 2010 & $2.1 \%(7497 / 350,729)$ & $1.7 \%(5898 / 350,729)$ & $0.5 \%(1599 / 350,729)$ & $2.2 \%(15982 / 722,212)$ & $1.9 \%(13477 / 722,212)$ & $0.3 \%(2505 / 722,212)$ \\
\hline 2011 & $2.4 \%(8687 / 360,208)$ & $1.9 \%(6856 / 360,208)$ & $0.5 \%(1831 / 360,208)$ & $2.3 \%(16774 / 713,875)$ & $2 \%(14178 / 713,875)$ & $0.4 \%(2596 / 713,875)$ \\
\hline
\end{tabular}

Infection Burden-THA

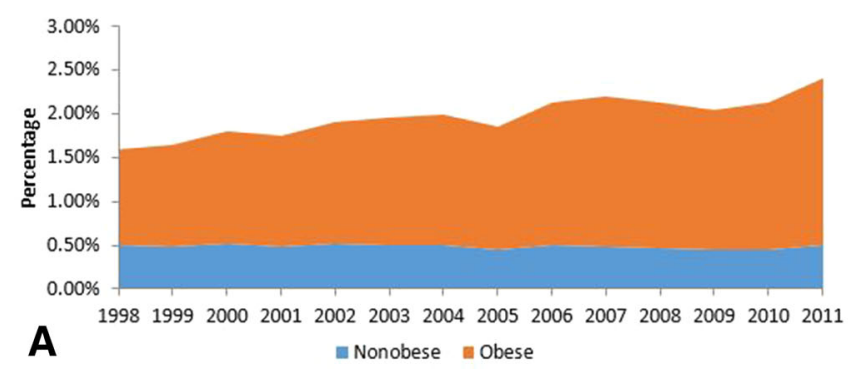

Fig. 5A-B The figure shows the annual trends in the infection burden of THA (A) and TKA (B) in the United States stratified by obesity. The annual growth in infection burden in obese patients was higher than that in nonobese patients for both THA (difference in

similar to previous studies, although separate analyses for primary and revision TJAs could have produced different results [22]. The infection burden reported in this study is obtained from a cross-sectional database like NIS and is different from the incidence of infection, which can be obtained only from longitudinal cohorts. The study is also limited by lack of recent data because the NIS database was available only until 2011 when we started the study. Finally, although an ideal approach for this study would have been to directly estimate the number of obese individuals undergoing these procedures from a national joint registry, such a registry is not available in the United States and the numbers reported in the study are crude estimates of obesity prevalence.

We found an increasing prevalence of obesity among patients undergoing THA and TKA. The prevalence
Infection Burden-TKA

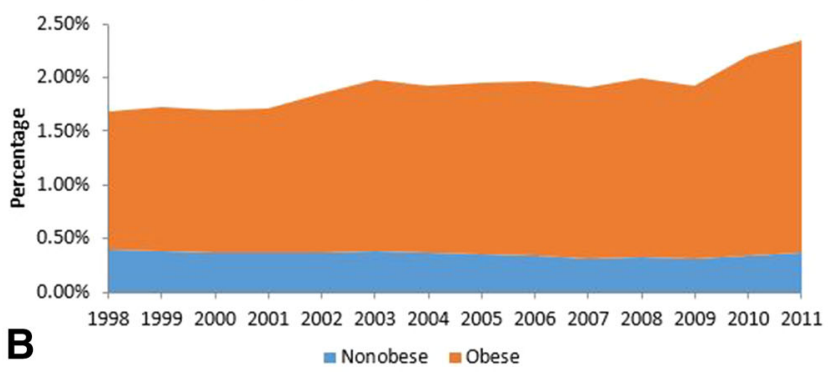

slopes $=0.06 \% /$ year $[\mathrm{CI}, 0.05-0.07], \mathrm{p}<0.001)$ and TKA (difference in slopes $=0.05 \%$ year [CI, 0.04-0.06], $\mathrm{p}<0.001$ ).

estimated in this study is higher than the national prevalence reported by Odum et al. [37] and closer to the prevalence reported in various single-institution studies $[11,19,28]$. For year 2008, Odum et al. [37] estimated the national prevalence of obesity in primary TKA to be $19 \%$, whereas Kremers et al. [19] reported it to be 59\%. Fehring et al. [11] reported an obesity prevalence of approximately $42 \%$ in patients undergoing primary TKA at a single center in 1990 , which increased to $60 \%$ by 2005 . This suggests that obesity coding in NIS data can be highly unreliable in addition to validating the methodology used in the present study. Although obesity was associated with the majority of the growth of TJA, the results of the current study suggests that growth in TJA was evident in the nonobese population also. This is in agreement with the study by Losina et al. [25], who demonstrated that obesity alone 
would not explain the growth in TKA. Although the methodology used in the present study is different from that used by Losina et al., our results support the fact that there has also been considerable growth among nonobese individuals. The increase of TKA (1.8 times) and THA (1.5 times) in nonobese individuals was higher than the US population growth of 1.1 times for the same time period, suggesting expanding indications for TJA [41].

There was an increasing prevalence of obesity among patients undergoing revision THA and TKA. The revision burden associated with obesity increased in TKA while remaining unchanged in THA. The prevalence of obesity among revision TJA reported in this study is similar to that reported by Kremers et al. in a large series of revision TKA and THA from a single center $[19,28]$. With obesity known to result in higher medical costs, even in patients without other comorbidities, the high prevalence of obesity is likely to result in additional financial burden to the health system [19]. Although obesity may be associated with a higher complication rate, the functional outcomes in obese patients are often comparable to that in nonobese patients and should also be considered while offering TJA to obese patients [31]. McLawhorn et al. [32] showed that functional improvements were higher in obese patients undergoing THA suggesting that higher BMI alone should not prevent a patient from undergoing TJA.

The present study demonstrated an increasing prevalence of obesity among infected THA and TKA. The infection burden associated with obesity increased in THA and TKA, whereas the infection burden associated with nonobese patients either remain unchanged (THA) or decreased (TKA). Although the increase in infection burden has been previously reported, the factors contributing to this rise are unknown, although some have suggested it may in part be a result of increased antibiotic resistance [22]. However, the resistance patterns were not shown to have changed with the prophylactic use of antibiotic cement [13]. Obesity is rarely an isolated comorbidity and often predisposes to other comorbidities like diabetes mellitus, hyperlipidemia, and atherosclerosis, which place patients at higher risk for infection and wound complications [10, 34, 37]. Given the strong association of obesity and infection, the present study suggests that obesity may be associated with the increasing infection burden of TJA.

The growing prevalence of obesity in the United States has been accompanied by an increase in the prevalence of obesity in TJA. The influence of the obesity epidemic was more obvious in the increased use of revision and infected surgeries. The obesity epidemic could perhaps explain the increasing infection burden and stable revision burden in the United States despite the advancements in the field of joint replacement. With the obesity rates projected to rise further, the economic burden of complications after TJA is expected to grow. Although obese patients benefit from TJA, surgeons should be aware of the potential implications of the growing obesity prevalence and efforts should be undertaken to reduce complications in obese patients.

\section{References}

1. Andrew JG, Palan J, Kurup H V, Gibson P, Murray DW, Beard DJ. Obesity in total hip replacement. J Bone Joint Surg Br. 2008;90:424-429.

2. Bourne R, Mukhi S, Zhu N, Keresteci M, Marin M. Role of obesity on the risk for total hip or knee arthroplasty. Clin Orthop Relat Res. 2007;465:185-188.

3. Centers for Disease Control and Prevention. Department of Health and Human Services. Behavioral Risk Factor Surveillance System Survey Questionnaire.1984-2014. Available at: https:// www.cms.gov/Research-Statistics-Data-and-Systems/ResearchStatistics-Data-and-Systems.html. Accessed May 15, 2016.

4. Centers for Disease Control and Prevention. National Health and Nutrition Examination Survey. Available at: http://www.cdc.gov/ nchs/nhanes.htm. Accessed May 15, 2016.

5. Cram P, Lu X, Kaboli PJ, Vaughan-Sarrazin MS, Cai X, Wolf BR, Li Y. Clinical characteristics and outcomes of Medicare patients undergoing total hip arthroplasty, 1991-2008. JAMA. 2011;305:1560-1567.

6. Cram P, Lu X, Kates SL, Singh JA, Li Y, Wolf BR. Total knee arthroplasty volume, utilization, and outcomes among Medicare beneficiaries, 1991-2010. JAMA. 2012;308:1227-1236.

7. Crowe JF, Sculco TP, Kahn B. Revision total hip arthroplasty: hospital cost and reimbursement analysis. Clin Orthop Relat.Res. 2003;413:175-182.

8. D'Apuzzo MR, Novicoff WM, Browne JA. The John Insall Award: Morbid obesity independently impacts complications, mortality, and resource use after TKA. Clin Orthop Relat Res. 2015;473:57-63.

9. Derman PB, Fabricant PD, David G. The role of overweight and obesity in relation to the more rapid growth of total knee arthroplasty volume compared with total hip arthroplasty volume. J Bone Joint Surg Am. 2014;96:922-928.

10. England SP, Stern SH, Insall JN, Windsor RE. Total knee arthroplasty in diabetes mellitus. Clin Orthop Relat Res. 1990;260:130-134.

11. Fehring TK, Odum SM, Griffin WL, Mason JB, McCoy TH. The obesity epidemic: its effect on total joint arthroplasty. J Arthroplasty. 2007;22:71-76.

12. Golinvaux NS, Bohl DD, Basques BA, Fu MC, Gardner EC, Grauer JN. Limitations of administrative databases in spine research: a study in obesity. Spine J. 2014;14:2923-2928.

13. Hansen EN, Adeli B, Kenyon R, Parvizi J. Routine use of antibiotic laden bone cement for primary total knee arthroplasty: impact on infecting microbial patterns and resistance profiles. $J$ Arthroplasty. 2014;29:1123-1127.

14. Haverkamp D, Klinkenbijl MN, Somford MP, Albers GHR, van der Vis HM. Obesity in total hip arthroplasty-does it really matter? A meta-analysis. Acta Orthop. 2011;82:417-422.

15. Haverkamp D, de Man FHR, de Jong PT, van Stralen RA, Marti RK. Is the long-term outcome of cemented THA jeopardized by patients being overweight? Clin Orthop Relat Res. 2008;466:1162-1168.

16. Hebert CK, Williams RE, Levy RS, Barrack RL. Cost of treating an infected total knee replacement. Clin Orthop Relat Res. 1996;331:140-145. 
17. Jackson MP, Sexton SA, Yeung E, Walter WL, Walter WK, Zicat BA. The effect of obesity on the mid-term survival and clinical outcome of cementless total hip replacement. J Bone Joint Surg Br. 2009;91:1296-1300.

18. Kerkhoffs GMMJ, Servien E, Dunn W, Dahm D, Bramer JAM, Haverkamp D. The influence of obesity on the complication rate and outcome of total knee arthroplasty: a meta-analysis and systematic literature review. J Bone Joint Surg Am. 2012;94:1839-1844.

19. Kremers HM, Visscher SL, Kremers WK, Naessens JM, Lewallen DG. The effect of obesity on direct medical costs in total knee arthroplasty. J Bone Joint Surg Am. 2014;96:718-724.

20. Kurtz S, Mowat F, Ong K, Chan N, Lau E, Halpern M. Prevalence of primary and revision total hip and knee arthroplasty in the United States from 1990 through 2002. J Bone Joint Surg Am. 2005;87:1487-1497.

21. Kurtz S, Ong K, Lau E, Mowat F, Halpern M. Projections of primary and revision hip and knee arthroplasty in the United States from 2005 to 2030. J Bone Joint Surg Am. 2007;89:780785 .

22. Kurtz SM, Lau E, Schmier J, Ong KL, Zhao K, Parvizi J. Infection burden for hip and knee arthroplasty in the United States. J Arthroplasty. 2008;23:984-991.

23. Kurtz SM, Muratoglu OK, Evans M, Edidin AA. Advances in the processing, sterilization, and crosslinking of ultra-high molecular weight polyethylene for total joint arthroplasty. Biomaterials. 1999;20:1659-1688.

24. Liu W, Wahafu T, Cheng M, Cheng T, Zhang Y, Zhang X. The influence of obesity on primary total hip arthroplasty outcomes: a meta-analysis of prospective cohort studies. Orthop Traumatol Surg Res. 2015;101:289-296.

25. Losina E, Thornhill TS, Rome BN, Wright J, Katz JN. The dramatic increase in total knee replacement utilization rates in the United States cannot be fully explained by growth in population size and the obesity epidemic. $J$ Bone Joint Surg Am. 2012;94:201-207.

26. Lübbeke A, Stern R, Garavaglia G, Zurcher L, Hoffmeyer P. Differences in outcomes of obese women and men undergoing primary total hip arthroplasty. Arthritis Rheum. 2007;57:327-334.

27. Manek NJ, Hart D, Spector TD, MacGregor AJ. The association of body mass index and osteoarthritis of the knee joint: an examination of genetic and environmental influences. Arthritis Rheum. 2003;48:1024-1029.

28. Maradit Kremers H, Visscher SL, Kremers WK, Naessens JM, Lewallen DG. Obesity increases length of stay and direct medical costs in total hip arthroplasty. Clin Orthop Relat Res. 2013;472:1232-1239.

29. Martin B-J, Chen G, Graham M, Quan H. Coding of obesity in administrative hospital discharge abstract data: accuracy and impact for future research studies. BMC Health Serv Res. 2014; $14: 70$

30. SMcCalden RW, Charron KD, MacDonald SJ, Bourne RB, Naudie DD. Does morbid obesity affect the outcome of total hip replacement? An analysis of 3290 THRs. J Bone Joint Surg Br. 2011;93:321-325.
31. McLaughlin JR, Lee KR. The outcome of total hip replacement in obese and non-obese patients at 10- to 18-years. J Bone Joint Surg Br. 2006;88:1286-1292.

32. McLawhorn AS, Steinhaus ME, Southren DL, Lee Y-Y, Dodwell ER, Figgie MP. Body mass index class is independently associated with health-related quality of life after primary total hip arthroplasty: an institutional registry-based study. J Arthroplasty. 2016;32:143-149.

33. Mokdad AH, Serdula MK, Dietz WH, Bowman BA, Marks JS, Koplan JP. The spread of the obesity epidemic in the United States, 1991-1998. JAMA. 1999;282:1519-1522.

34. Must A. The disease burden associated with overweight and obesity. JAMA. 1999;282:1523.

35. Nationwide Inpatient Sample (NIS). Healthcare Cost and Utilization Project (HCUP). 2000-2011. Rockville, MD, USA: Agency for Healthcare Research and Quality; 2013.

36. Niu J, Zhang YQ, Torner J, Nevitt M, Lewis CE, Aliabadi P, Sack B, Clancy M, Sharma L, Felson DT. Is obesity a risk factor for progressive radiographic knee osteoarthritis? Arthritis Rheum. 2009;61:329-335.

37. Odum SM, Springer BD, Dennos AC, Fehring TK. National obesity trends in total knee arthroplasty. J Arthroplasty. 2013;28:148-151.

38. Ogden CL, Carroll MD, Kit BK, Flegal KM. Prevalence of childhood and adult obesity in the United States, 2011-2012. JAMA. 2014;311:806-814.

39. Singh JA, Lewallen DG. Increasing obesity and comorbidity in patients undergoing primary total hip arthroplasty in the US: a 13-year study of time trends. BMC Musculoskelet Disord. 2014; $15: 441$.

40. Suleiman LI, Ortega G, Ong'uti SK, Gonzalez DO, Tran DD, Onyike A, Turner PL, Fullum TM. Does BMI affect perioperative complications following total knee and hip arthroplasty? J Surg Res. 2012;174:7-11.

41. US Census Bureau. Health Insurance Coverage Status and Type of Coverage-All Persons by Age and Sex: 1999 to 2012. Available at: http://www.census.gov/data/tables/time-series/demo/healthinsurance/historical-series/hib.html. Accessed May 15, 2016.

42. Wagner ER, Kamath AF, Fruth KM, Harmsen WS, Berry DJ. Effect of body mass index on complications and reoperations after total hip arthroplasty. J Bone Joint Surg Am. 2016;98:169_ 179.

43. Werner BC, Evans CL, Carothers JT, Browne JA. Primary total knee arthroplasty in super-obese patients: dramatically higher postoperative complication rates even compared to revision surgery. J Arthroplasty. 2015;30:849-853.

44. Wong JM, Khan WS, Chimutengwende-Gordon M, Dowd GSE. Recent advances in designs, approaches and materials in total knee replacement: literature review and evidence today. J Perioper Pract. 2011;21:165-171.

45. World Health Organization. Physical Status: The Use and Interpretation of Anthropometry. Report of a WHO Expert Consultation. Geneva, Switzerland: World Health Organization; 1995.

46. Zhang J, Yu KF. What's the relative risk? JAMA. 1998;280:1690. 